Supplementary Table S2: Full list of predicted GIs in UM_CSW genome.

\begin{tabular}{|c|c|c|c|c|c|c|c|}
\hline $\begin{array}{l}\text { Genomic } \\
\text { islands }\end{array}$ & $\begin{array}{l}\text { Genomic size } \\
\text { (bp) }\end{array}$ & $\begin{array}{l}\text { GC content } \\
\text { (\%) }\end{array}$ & $\begin{array}{l}\text { Contig } \\
\text { number }\end{array}$ & Start & End & Gene description & Gene-sequences \\
\hline $\mathrm{GI}$ & 11040 & 62.3 & Contig 2 & 63446 & 74485 & hypothetical protein & $\begin{array}{l}\text { MTQTERLPAYTEAHPPTPSSDELRAQIPGWGADLDPKDRP } \\
\text { SNPKLRQDLPTETHWDFPERQPEKWPRERSVEHRFLTPVF } \\
\text { GTAQPPSGVSGMLRRYAYKFSEGRAAHWLILLYADRVDTA } \\
\text { EHHVRSFLTTRPDNPITETGIKSELTHHGIQSRLGRKRSDLVH } \\
\text { IWMDPFIVAGPWILGGQGIASLARKAVKAVGNNGGRGGG } \\
\text { R }\end{array}$ \\
\hline
\end{tabular}

low molecular weight T- MTGADGAVSTNPEPQPDQDWIVNTKCSFPQFVAALNAED cell antigen TB8.4

hypothetical protein IRAAQQFAITPAAQSWVGSYLASSPDQRRQMLRRAQSIPD ARQYIDTMLRVASTCKNF

MRYEEFISSVAERSGLFQGDAVALTRATLTTLAERISGGEAR DLAAQLPSPLQDALLPTEEEAEAFSFDEFVNRVAERSGRDTE AAEAAVDAVLATIRDAVTPGEFDDVLSQLPSEFQRLGTR

protein of unknown MGPPGRRFDWHSPFFVGLTASAGVAVTYGAVRGLAAVSS function UPF0118 VLLLIGVAFFIALGLEPAVSWLVGKKVPRWAAVAVVLFAVIA VVAGSLAAAIPPLVQQAREFTDQAPHYIQHIGDHSSWIGRL NDRFHLQQRITETVHGSGGAVVGQALKAGEAVFGAVADV VVVVVLTVYFLVDFPRIRATLYRFVPHSRRPRAILIGDQMFA KVSAYVFGNIVISAIAGVATFVWLVIFDVPYPLLLGIFVALLDL IPFGSSVAGVIVAAVALTVSIPVAIATLLFYTLFRFVEDYLLVPK IIGRAVNVPAVATLLAVLVGGALLGIVGALVAIPIAAAIQLAA RELLFPTLDKL 
hypothetical protein

hypothetical protein

hypothetical protein
MVVIGHIADPEEPRHGRRGKAVQSTIARLAAAMVGTAGIE DALSKLTSASLALIPGADCAKISTIENGHLRSITATSQLTSSLDS AQQAAGQGPCLETISAKKAIRCDDLRTDTRWPRFTPRATTA GVYSVLSTPIDIPGETGATLTLFGFRAEAFGPDAEAVGAMLA NHAAIALMHVKQERQFKAALATRNVIGQAKGMIMERFGID AARAFAMLRAISQQTNTPLRELATRLVDGAKR

MQRLRAGTSIIYASGSLDGETRAVLCRAVADELTQGSAQLV LELSSATSIDDTAVEALVDATGLAAESDTSICLVTSPTGPIARA LAAADLLERFEIFATVDEAQRHP

MGIAHRAEPPSLHSVFNLSPLLFDGRRADAVLELAAEAVRSL GKCSSEGAYRVVDGSLVDNSDPDRPLDSGLDAAVTASVGV DHEIVLPDSNWRYAMTLRTTNTVTGVLVVRADNPASCHEL VLLKMLAQQAATAMASADTIERERRQHVQLRELTDRHEETI HRLSRSVAELERREHIHKALTNLSGSADAASIADALHDLTSLT VSVEDTFGNLRAWSPAPIPTAYRAVGGGNREDVIRNAGSR GHHSDCGNRIFSLIRARAELLGVVVLHDPQRRADRLDIFALE YAAAVLAVEFSHQRSIAETESRLSRDLVDDLLTGTDNATAYA RGEALGYNLHRPHRVTVLQWSAEIDGALIARSATRWATSA GLHPLCARRPSMTILLTEDLPQPOSLYRAISAAVGHGRGWI AIGSVATSPSELPRSFAEARRTLRMQKASVGRHGFRRFDDL GVCRILDPSDNGPEVGEFLAEWLGPLMAYDQEKNAELVNT LARYLDSGGNYDQTARALNIHRSTLRYRLGRIRDVSGRDLQ NVETRLNLHLATKVSEMIGETQPIMRAASTGERRPSDEGM PK 
putative transcriptional regulator, AraC family

hypothetical protein

hypothetical protein

hypothetical protein

ThiJ/Pfpl family protein

hypothetical protein

This/Pfpl family protein
MTELPFDLAPEVRTTDIDEAASALGRVYVTAELVPNKTNSV GMHMNAAQLPLITAGYLGFGADVTVRANDVTAYYIDAPLS GSAVNRWRDGELVKTTTGSVAVFTPGTPCVLDWSGDCGQ CLKVSEPQMRRQLEAMLNRPVRKRITFARQFDLSTTAAHD WYHLVSLLAREVGRTDGLLNHRLAAANLQLLLIQGLLQIQP HNYTEALAESEGAASANVAKRAIDLLHAHPETMWSTAQLA RQTGVSARALQRAFERSDQPSPMAYLRRLRLHRVYTELAAS SPDSVTVTMVAGRWGFVHLGRFAGQYQQLFGETPAETLR HRVADALPTQPQPPL

MFISDRTTPQEPDMTTADEDSERDFLAKCVADAYQMLEIIP GVNPNGAALVWLADQFMQVRRRTESHLASTA

MKRPNSRFLLAISRTGWLGRTVEILLRGLLASDTGTVKVSMP WSI

MWSEYYDVATECAVTLRMLLASMSNVTSIWGSPRGIGRM LPNFETAQ

MPNMLQGKTIAILAADGVERVELVHPSAAVHRAGGQTELL SLRNGWIQMRNNDLDPAGTFTVDRAVAGASIDDYGGLLLP GGTVNPDKLRTDERAVAFVRGFVESGKPVGVICRGPLTLIEA GVVAGRTLTSYPSVRTDLRNAGANVVDEEVVVDGNLISSRS PDDLPAFCVAIVKAFAKTPAGTS

MAQDTENQHTTGTQTGTENQGTTENDSAGSQAPGGNPD NRETVQNMDAPELRTAVIELLTIIDDSDEMDVVVTISDDGT VVVEAA

MTHELRGQRVGILAADGVERIELEQPRGALHGAGAMTEVI SLHPGEIQARQFDMEPAGSFPVDRLVGDVSADDYDALLMP GGTVNPDKLRMNADAVAFVKAFADTGKPIAVICHGPWTLA EADVIQGVRMTSWPSIRTDLRRAGADVVDEEVVVDRQFISS RSPNDLPAFCGAIVVQFAKAKQPV 
hypothetical protein
61.7
14745
Contig $17 \quad 84910 \quad 99654$ hypothetical protein
MATKALLVRLEAKPGKEDAVEKFLLSALPLVEQEPGTKPWF AVRFSPSIFGIIDAFPDEAALEAHLGGAVGKALAEKADELFAS PPDISKLDVLANKL

MPGITRSSAHSTSSIAERLTLRPDTTRTASRNTDGAADAGRP

RVPEAGKAAGRESGSGRSDGGSGVDRVPAGTNGLVICAKS VRDTVDGIVDAELSASDALGLRFPRPNMVASAAALFEPAEP WAHGRDRLDVEPECGRFRITIGPGVVRLGWTNPVRAEKAS ERTVGQHMQDVAGEVDRIKAGRDVPDPSGRAITEWSRKS RAAMCRTFAELDYTPMVESGRVPAMVTLTYPGDWESVAP DGASVKRHMMLWRKRFQREWGEPARYIWKMEFQRRGA PHIHLWMAPPYTVGRSGRRFSEWLSQEWAEVVAHPDPEQ RTRHLRAGTAIDSLNGLRACDPKRLAIYFTKHSSPNRLGDKE YQHIVPEAWRRPGRGPGRFWGVHGLERATAVVEIAQDAY LTARRIIRRWSRSQAIHSDCTHCFPTALVPRMTRVAVQRTN SKTGKVRHRNVCRRRLLCRQGGFAGGYALVNDGPAFASQL AAALSADRAKAVRDEQLAGRK

hypothetical protein

MHRQPQLLNDCVHLTSLFSVGKSEFIKLPTIFMILQPQE

Transcriptional regulator, MREVPRKIADRLPPAAALFADRGLNDTKIEDVAATTGIAKAT TetR family
LYYYFAGKEEILAFLLEDVLQQVAHEVAAVVEAEGSAAQRLH AVISAQLRVMAQRPAVCRALIGELGRAARMPVIADMISAAY FAPVETLLRAGAGDGSLVTLDNPAVAAIALFGAVTTSALTYL VLDDALDEDRIARTIHDLVFVGLRPR 
putative cytochrome P450 hydroxylase
MTTVQLRYDPFDATIQDDLYPIYRQLRDKAPLYRAADSNTW VFSRHEDVVSALLDHHTYSSVDGIFPTPPGSDFHASFLPMMI LMDPPRHDQLRALVSKAFTPRRIAALSSGIEDLAEDLSDCLD RGAGSVDFAADFAAVLPAMVIADLLGIPREDRAQFRQWST TMVQSNPARGEMGEGLAAAAAVYAYFTDFLAERRREPRG DLMSALVSAEIDGKRLTDEDLLGFCLLLLIAGHETTSNLLSNA AVVLASDPDIRRRLVADDNLLGPAVEELLRYDSPVQGLSRTL TRDVTLHGVTMSVGDSVLLLFGSANRDERVFADPDVFDIGR KPEHQVAFGRGIHFCLGASLARMEARIALRALLARVPNWEV DLENAQRLRSGLIRGYMSLPISWSAN

Transcriptional regulator, MERDGFDMPVDDDVDPRLVRSRTRLLDAATKLLSAGGIEA TetR family

VTIDAVTKASKVARTTLYRHFSSSTQLLAATFERLLPQVHLPP ATGSLRDQLIELLSRQATLFQEAPLHVTTLAWVALGPTSNST QETYDRHALRTRIIEQYRQPFDLLLQSPEACADLDEFDPELIL CQLVGPVAFARLTGLRAIDRQDCERIVDDFLAAHRRKVDEP 
MADNGWVSSGVATLSRPVREQLRKVAAGEGAYSDRLARL AGFSIRHKVLVIGAWVVTAGVLAMLFPQLETVVKQQSVNL VPPDAPSLQTVDRMGTAFGEQGSKTTVFVAFEDPTGLTAP VRQRYKTMVSRLRADSQHVRLVQDLLADPVTAGQAMSQD GKAWYVPVGVAGTLGDPRAAESVRAVRAIVAESFSGTSTIV RVTGPPATFSDLIDSAEQDLIGISIVTAGLIAVILLVIYRSLVTAL LPLLVIGVSLAVGRGVLSALGESGMPVSQFTIAFMTAILLGA GTDYSVFLISRYHEQRRQMISADLAVINATATIGRVIMASAA TVAFAFLSLVFAKLSVFSALGPACAIAVFVGVAATVTLFPPVL ALAAKHGIGEPKADRTRRYWNWIAVAVVRRPAPLLAASLAL VLGLAAVALTMHISYDDRQGQPATTTSNEGYRLLDRHFRKD IVITQFMVVESPTDMRTSKGLADLDEMASRVSQLPGVTKVS GVTRPTGARLDQAQLSWQNGQIGNKMAGAVAKGDAHKD DLAKLTSGADQLAGGLAQLDTTLRTALTPLTAVLTQAQSSG SQFQHFRPLLQQLSATTPTIDQAIRTGPGLRQEAQQAQNAI ATIDPLVGALNTSPWCATTPECAQIRDQVQILVTLRDGGFFS QLANLGDMYQPGSDNAAGTVADLQSTVTSLNKAFGALGD PADMAGNIRRLQNGISQLASGAQALATGVHTLADSNIEML SGMSQVATQLQNSARSSAGSDNASGFYLPANTFENRQFAD VAKHFMSADGKTARFAIESSYNPYSSQAMDLAQKITEVAGA ARPNTSLANATISMAGFPAVNSDIQRLLSADFHLLAIATLVIV GVILVLLLRALLAPLYLLGTVVLNYGAALGIGTLVFQYGLGKEI AWPVPLVAFIILVAVGADYNMLLISRLREESAHNIRVGVLRT VANTGSVITSAGLIFAASMFGLMVGSVGIMIQVGFIVGCGLL LDTFVVRTLTVPAIATLLREASWWPQRKSSTHNGRPHRTT 
Hydrolase

hypothetical protein

hypothetical protein
MADCYRHAATHPVVLLLHGGGQNRHAWATTARRLYSHGY TVVAYDTRGHGDSDWDPSGQYDIERFVSDLISVRGHVSAD SPPAVVGASLGGLIILATHLLAPPDLWAAVVLVDITPRMEFH GARRILSFMAGHPDGFGTLNDAADVIAEYNPRRARPENLD GLHKVLRQRSDGRWIWRWDPAFISSNFDVLQGNLMTGSE EFDAISGFLAEGARRITAPTLLVRGALSDVVSOETVSEFRQLV RHAETTDVTGTGHMVAGDNNDAFTAAVTDFLDRAMRTLT

MGLTDASKGAVMHTQWIEQCTVQRVSLHEGLILDLDGYNE LVISRPLRLTLPPAGAWPSDEVLIDPINLSPEERPLLDLAGAIC TRAWCDDDGALHLCFSPGHRIDVDPDVAATSWELYGKCH GYVACLPRGRVRAIRHDLPVDENDSDATQPKAMAHQ

MAITIPSGRPNWRFMRYVRPPTRDSKLEPLYPLRPATRPVR LGIDVGTIAEPPEEGYITGFLTRDEIEVHLLIPAATEAPSGWTE LLDEPPCHTVNFTNVADAGKFCDAAEFSVSTARGESYRAWS KARFFAAYQQLDEHDAPDGLPPLTLDQRHRAAAYAAAAGA VGIDAIVTTAPTAGRTDVADNDVVVSVTPDAAVPLIGHYLR VTSNPVVTVERGMLVGGGSWETTESTATIVNLYDWGTVSG LPYFDAASMFAAAAKGGPEAAEAFTSVRIRLRRAARAFDDL LAALSNPLDGKRNEDVAEATAEAFDRELLYLAAVFDIFGRAY QAMVDPSVDRKKARGSLDSRTFIDKEVRTQYDQSLLGDVT RLRVYAWLCKQLRNHIHDGVLAVDTHPGRSYGNTMNVAL NLSVIPELALGADNEMTQHHYDALGVWQTEPVSPFTGSSM VADLATTGFTLIRAALEYIEAFTKLIVRNKPANAPSSSAFLGCV QARPGEVEPAPPKRAVFYQALFGLHPDSV

MAAVDQSDNPMGQVLVNAGETFYLHRQSGFLKDFAPNTV LKRFA 
Transcriptional regulator KorSA, GntR family

FIG00831412:

hypothetical protein

Transfer protein traSA
MLQLGQIDRADDKPPYRQIAGMLREAIRSNRLAPGERLPSE TELIEHFGVARMTVRQAVQELRSEGLVVSQHGRGVFIRPTP PIRRLASDRFARQHRAAGKAAFTVEAEKSGYSPQVDNIAVS REKPNSFVAERLRLSPDDDIVVRSRRYLANGRPVETAVSFIPA AFAEGTRIEQVDTGPGGIYARLEENGHVLGHFTEEVAARMP TPEERRQLELEPGVPVLTVLRTAYDANDVAVEVCDTVKVAS AYLLEYEFPAR

MSVPKWLKVSHQQVFPRKAFVVSDVTAVIDYERSTKDNKV QATDRESGAPLWQVEVLDGDPTASKRARTLTVKFATPKQP VCPPNTAGMPFTPVIFEELYILPYVDRSSESGRIAWSFRASG MTADTGKAGSSAGDRVSA

MSPYDPVVPSDPYSIPDPDAGIDFFDLTHLLAVGAMWLIAV IGVVAVSLVVWRVRSPATFGRYVATPACRARWLIWASISW PRVAKACGLSTPEHVTRTDAQGKSRTRTVWTHPRLLGVSM FGDCLRMTVRTRTGQTVDDLENAVPAIRDAVGAHSARSTLI GPGTVRVEFVMKQCLSEAETAAFPTSVEPTGVEVGRRENGS AWTLRVAGLHTLTVGCSGAGKGSVFWGIAGGLGPAIKAGT VRLFAVDLKYGIEVSVGSALFSGIATTEANAARLLTNLEELLDS RGRRMAGRARSHTPSTAEPLVVLLIDEFAGLTAYMTDAALR KQVAGSLSRILTKGRAVGIVVAAFMQDPRKEILPMRGLFTQ TVALRLRSRDEVAMVLGDGLADAAPAHRINPNEPGTGYVIA EDGSTMRVRADFWPDSLIRSVAQEYGPTSSSRAGSAEN 
Coenzyme F420dependent

oxidoreductase

hypothetical protein

putative cytochrome P450 hydroxylase TetR family
MAKLSVATPVVMLPAANGSWEKDASIEDLAQIAEAADRLG YHHLTCAEHIGLPAAERERRGTRYWDPLATFGYLAARTQRI RLTTYVLVLGYHHPLEIAKRYGTLDKVSNGRLILGVGVGSLKE EFELLGASFDDRGARGDDALRALRASLSVPEPTYHGEFYSFS GMVVDPCAVQDRVPIWIGGRTLRSLRRAVTLADGWAPFN VSPQQAREWLGRFDIQPGFEVLLPPLAPLNPIDEPDRTRHII ADTVAHGATIVSTMFRHRSLQNYLENLHALAEIYPPDGGAA

MNRVKAGVFSLTPPAPADDDGSYLRWHLLDHMPEQYQLP GIVHGLRWIADGDYADHRLAANGPLSQIGNAVHYLVSDPV EETLDDFVALGSALRENGRFPISRPYLQVAGLRLLQWHSAP QALISGEVVPFRPHRGILLIVEEPADGRSNEWLQWLHAEHY PALLATPGTVGAWTFGTSTGWIHLPAGWRTDQQYITVVYL DADPLATTDAVAALVEDRWRSAAVRPVFAGPLRTMISWD AWPTS

Transcriptional regulator, MTDQEPRLRQRTDGRLNRSRDPAILNAALAALAEHGYHAT NMDDIAARAGVGKAAIYRRWSSKAALMTDALVYWRPDLL SADVPDTGSLAGDLDALVERIKRSDSDLVSNDLVLRVAMEA AHEPELASALDDLMLGKGKRTMTAILTHAAKRGEISGDRD WSLVADVLVAMGLLQVARGQTVDANFVRQVIDTLVLPAV RTTNNA

MDPAMFFGTDAIQNPYPLYEQMRAASPVHRIGDSDFYAVC GWDAVVEAVARVDDFSSNLTATVVFEGDTGSVEPYSMMP LGDPMHSLATADDPAHAVHRKILLPHLSAKRVRVIEEFAQE TARRLLSESLVDGRIEWMSTVANRLPMMVVARLLGLPHDD VDTLIRLGFATTTLLDGLVTPAQLEAAGTAVMELSAYVLDHF DKAATDAQPGLMSDLAARCASGQLDQPVALSMMLTLFSA AGESTASLLGSAAWILVSRPDIQEQVRAHPDLLGPFIEETLRY EPPFRAHYRHVRRHTALAGVELPADAHLLLMWGAANRDA AQFESPDEFRLDRGGAKGHVTFGKGAHFCIGAALARLEARV VLGILLERTDWIEAAEIGEWLPSILVRRLERLHLATR 
Transcriptional regulator, MAGVDWLVGQDRGSEATARIHAAAADLVSERGWEGFTIG TetR family ALAAKVHCSPATIYRHAGGRTAIRNAVVNIHAARVIESMRD AIEGLTGAERVVSATAIALQRIRSDPLTQMIRSTRPPIDDDW MPSSEVVVQCAQEVLGQHDPDPLAQQWLIHVFLALWIWP MKDAEAELQMLQRFLGPPYDAAGS

hypothetical protein

MMQGDLEIAYEIIGEGEPYILTGGGRFSKDAGGVRELAEAIA DRGKKVVIYDRLNCGASAVCFEGSSESVMQAESLARLIVDL

DLGPTVLLGGSGGARISLLAAARHPEIARAVVMWWPSGGA FGSMSLAEVYNFPSIRAAWNGTMADVADDPTWAEVITRN PRNRDRFLAQDKQTFIETMERWAHVYCACGNPLVAGLTDE EAAAITLPVLVFRSGKSDYFHTRATSERLAANLQNAEIIDPV WGDREFLDRYEDSRDNGVSVWINWKQLAPQIVDWTEKVL $D$

L-carnitine dehydratase/bile acidMIKVMEGVRVLELAQYTFVPAAGAILADWGADVIKVEHPV inducible protein $\mathrm{F}$ RGDTQRGFLYMGGIKLEADRHPLIEHPNRGKRSVGVDVSTP GGQEVLYELAKTSDVFLTNYMPQARQKNKFDVEHIRAANP NIIYARGSAYGDKGPERLVGGFDGTAFWTRSGVGHALTPEA LGGALPQGIPAFGDSIGGMNIAGGISAALFHREKTGEAVEID VSLLSTAWWAAGASVTQGMETGETMRSTMPDETPPIFNP FLGNFLTSDGGTINLCIVSPTGYIRDTFEHLGLPELADDPRFS DVMPLIRNAAEAAELVAKAIRGKSFDYWREHLKTMKGQW APFQSLLELASDEQAIANDMIVEYEASAGDKPFRVVRGPVQ FNHEPLETTRAPQASEHTEIVLMELGMEWDRIEALKDAGAI A

hypothetical protein

MDDPVTDVEDTVESFPGIESGGKSTEVGHFMAAMRRLQD VVVSTDPDNELWGSAAREVNDLCERFELHRVPAGFGLRGR GPHLPGLGHPLMPPWMMTEYGPDGVTMQGHFSOYHVG SNNAVNGGVIPLLYDWQFGMIVSAVDRRDSRTAYLHIEYRK VTPINQPLTSRGRIETIDGRKVFVTATMTDSDGSVLSEASGL MIQLKSNQP 
Long-chain-fatty-acid-CoA ligase (EC 6.2.1.3)

hypothetical protein
MYPGHWGITKPDHPAVIMAGTRETITYGELDRRSRCLAGFL WSRGLRRGDHVAILMHNDVRYFEMLWAALRSGMYVTPV NWHLKPDEVAYVIGDCEAKLLFTSARQSDLVEDIDWRTLPA LTTRVMADGTTTGFEPYESVVAFEGEPAEESSGAAMLYTSG TTGRPKGVLRPLPDTKPGDLDAASHRLAALFGIDEQSTYLSP APQYHAAPLTFTLAAHRAGATVVMMEKFDARDALKAIDM HGVTHSQWVPTMFVRMLALSPEDKQAADLSTHSVALHAG APCPPEIKRQMIDWWGPILVEYYGGSDGGGFTVIDSLDWL AHPGSVGRSRTGAVYIVDEDADAVVGPGQTGTVYFSGDPG FVYKGDPEKTERSRRAGGELSTMGDIGYLDADGYLYLTDRK EFTIISGGVNIYPREAEDVLILHSDVQDVAVFGIPHHEFGEEV KAAVQLKPGVTATPELAGQLIAFTRARLASFKCPRSVDFLEQ LPRTPTGKLRKADLRSAYAQGNSTP

MANKMFRKYGYDAVTLEQIADACVMSVRTILRYFNTKEAL ALAGEHELLESFRAQLGVRETDAVTCWRNFRNETLPLMETP ESRERMRAIYETPPLLAEFLRIGEGYQQCLTAAIAEDWGEVA SLEATVFASVLVSTTSAAFRRWYVSEEPFDLVALQDIVDRVC VAFDKGRQSADGRRVGRVSAANTAPTRRAPTGRR

MKMYAARILSIMRLAGGISSPALGRTNFTSSSKRSSFNSFQI WILPPGKVL

MREGFSWATATDSGRGSQRRSWAAAVDIFVDMAAADGG AATSAHFSATRIARLAISIVQVWLYEWWHGPRDSAIEEDAE RLVSFLSLWLRDED 
hypothetical protein

hypothetical protein

Protein of unknown function DUF1486

hypothetical protein

hypothetical protein

thioesterase 4
TesB-like acyl-CoA

MYRWVAAVMTAGYIVTAAGTGLKIFPTTPITGLVACIIFTNA IFVAFWACWFLEGPGKKRTTLEKADEFTMIWACVAAVGSE MTWELPWLVADALGRAHITPHDRWVYIWYYYARVDERYLI SDGALWGMEAVVILAAVLLLVOWFRLRSAPTHDPKRISAL WWSLLAMVVMLTIFVVYYAAEVRHGFANVQRGFWDITVI FVYENLPWLAAPIISIPLTAKQLAYLYREQGRRTALAEEGALA DDRADVRSLV

MHASAPSRKVADLAERLQTIHFGREQSAEGGTIVSPSARIG DC

MTIFKQEIKAAMTPDMTTDSALRRSREELVLRHVAAENAH DIDAAMATFTHPRYEIMPTGMVFDGAESVRTMLLQQWA QLPMLQYSAEAIYHGEHGLVVETRTTAPGRSIDMLSMNLFG FRGADLVLERCYFDQSLLAAQLEALAPD

MDGPSALRRRRFKFTETPVYGTDDRSLGLRPVGVPAEASM QQ

MTRLTRNGSMISIEIDPTRVSMRSKGGATPSEHRRWGVRM LGPLAHSLSRRRIHE

MASPQEPYFTENGKTFVPSDVARGGWGPTVGGHAIGGLL ARAVESERADETLIPARFTVDILRRVALAPVRVEASVVRSGR RMQAVDAVMTQDGELVARAATLFLRQSEQPQEIPWTPTR SMPPLPEELTALPKGVPMQIVPYNDGVEMSGQLPWQQD GLRQAWIREIRSLVGDEELTPFIRAAMAVDVTASMTGFTRT GLGFINADYTLTLCRLPKGSFVGLAGLTHHSAAGVGVGAAT LFDAEGPIGTGVTTAAANTTFRGGAL

Transcriptional regulator, MRRSNLNAVARRANVSRSTLYRRFPTKEELLYAVVGRLTESI TetR family
FAELQHHTTGMTAKQLVVEVFCIAVRETTSNPVLHQLMVA EPDTLAALLGFFGPGMSAVLDRAVELAVAQAINAGAKMPP ADLRIVVELMIRLTTSLMNSPSAILDADDPAAARQFAQKFFA PMIW 
PROBABLE CHOLESTEROL MGRNLILELLAQGHQVRSLDLTRNPDADSRIEVAIGDICDPR DEHYDROGENASE VVAEVVDGIDTVFHTAAVICLLGGRAATSQYREHSFAVNVE ATQSLVSHARDAGVSRLVYTSSNTVVMGDEPIVGGNESLPY TTRFADLYTETKVVAERLVLQHNGSGGLLTCAVRPSGIWGP GDQTMFRKLLEAVASGQVRALIGSRKTVMDYTYIGNLVHA EILAANQLGPNGRASGQAYFITDGEPVNVFEFARPVVEACG QHWPRLRVPGGLVRAVMTWWQWLHFRLGVTAPPLEPQI VTRLCVDNYYSIDKARRDLGYEPMFTREQGLASSLLYYTDM FKTMKHQAG

hypothetical protein

hypothetical protein

hypothetical protein
MQNFNRPVAAGRKRVYVAMFVLGCLVTGIPFFGVGFGLM PPEPTYEIWGALIYIPLLVPLFTAWRDAPGENGTWTERAAEF TMVWFPVTASSOITWETPWLIGDLTGVMHGAGPQDRWV WLWWGYGAADTRYLTSDAGLYGMEVVAVIGGLALFTAW FYLLRAKHTGDTMKRIKGLFVGIVACSMMLAVVTIYYVSETR AGFRNLEQGFWEGFMFKFIFMNIWWVFAPLITIPFMIKQID YLYKSMPRSIHQDVQPGAELQHAV

MPNRRSDALPGIRGARREMYRQQILTAAEYEFARTGFAETR VATIAATADVSLATLYKNFAGKDEIWNALVEQRTNELVGLA RAATVGVQSPLERIIVGARAQVDFFAQHPNFLRLNVKENW SWATATEAARGGQRDAWRVGIAVMVREAEAAAASGELR HLRPQIVAGLAVSALQVWLTDWVNSGVQRSPSQIADELQD YLRRLLVVPRATDTTPLRD

MVRATGVIACSVFTIPMMIVFVTAWRDAPGENRTLLERAN EFQTIWFFAAAAGSEITWETPWLIGDLMGWMHLTPKDTW GFAWWYYGDVDQRYLTSDGGLWGLELAVVALAVILLVOW FRLRSAATHDPKRINALWWAFFAMAGMLSLFATYFLMEVR HGFSDFKLGFWEITVILGFENLPWLIAPVISLPFVAKQLTYLIR SAPAATGTPLRSGAVSSSAAGL 
MNHINIVVEEFDAAVMHFQRVFGAQLVLDLPQPEWHACL LDIGDVLFEVFSPPQFLLNARYGPHYVGIEYQTGMDAARRA VTMRNIRLVRDIGHAIHTHPADTLGIAWEFFDGDFRVMPN LKWLEPLHSPERWRAHPIGYTGLRRLSVAVHDCDRAAQFLA DFFGAMIVYKESRKSLSATIIGMLLADTTIELISPTGEGAISEHL HRYGDGIRSTVFEVADLERTHAHFKAIGIDLVDGDGPSTLAI EPRDNLGVMMEFCAS

Transcriptional regulator, MVNLLAGLRGETLSISEIAGQLRFNLSTCHSMLAELVKAGVL IcIR family

VRHPGTKRYALGPTLVTWGVASAFDSYRVLEFAEPEMSRLR HQLHVSCVARALVGPDTVVLARRDTDGPIAAFTPVGYRLAA VPPIGAEFIAWESDATIDEWLNRPQRALDTTERTRMHALLA EIRRDRYRFLWADPAEQTPLATELIQTSASEDEPGGDRLFEL LQRRGYGRGPRTGTLSAITVPIFGADAKPVLALLLTFNPVPID ISEVEHYLRPLIHTCQRITTTIGGRAPN

MPVAIGNPCVEEHVASENVESIETLRRNRVGRRARTSEHES DALE

hypothetical protein

MQFSQRRLAVSRWGGCPTYWSTKFIASLEIRHKSPFEQ

hypothetical protein

MFWQKVMINVACVEGQDPDVFDGGRVIAQSRSNRKGEA LHHLVGVRLVLTERESD

hypothetical protein MILDLIQTVAAVSVPIVVAVIGYKLNHRLKLYEASQWRNQEL IKARLQYFGQLAPMLNDLMCYLTFIGRWKELTPPDLIAIKRD TDRLFYSVAPLFSLAAVTAYQDFLGACFTTHTGWGTDARICS GFVRRREASREPWLAEWGQLFTHQDGDAIPESSMTAVRA TYDKLLATLVEDVELLQPRDRYADSNLVANAR 
hypothetical protein

hypothetical protein
MDALEGPSKISDSRQVALPKRLMDSLGWDKGDYVMFRLS DDDPEVMKVVLESVVLRQLRRGEDAERTMREQRAETTTDE SPQDRG

MTGLVRDPNVGFFDDQLTREAVVKLAACNRLTFFVGAGAS LDLNLPTWSALVSDLLKDTISANFGPDSPVETICAELVNQLF QVPAASVIDSLLFDEARASLSGRPARSTIHAKMLSTRNERLR KSLYKRDRFGDITVGPTLVGKMLELAILLRRAGVDVHIVTTN YDNAFEEAALHEPLFSIMSTERLRLVLFAESPPEAGDLDEGDI PVVHIHGLLRAPSGYAAAGHNPIEGKIVFSEVDYIDWETGDF RTYLTNRVQSGGLLTIGASLRDNNIVARLRDGTRSNNEPRYA LMPSESEFKYLSDKNIAEKYWMPFVEMAARRGFIFGVSILRP DFYGQVFQFLQELGVNVSSRINPGTAYVEYRARIRQWAVA WSTVRESDEPGMRGNIETACRHLAVKFEALPQFDHCKVEV WARCEPDDRTMRRWCSSQSTWRPDAWPHTTLIRPLSKQ NAVMAFSSRGTVINKTPGSADRRWTHCLTVPVFLDGEPWL GLPVGALEILLHESGAATSDECLNYLRANATPWVKEISKLGE KILTPEPWVD 
ATP-dependent DNA helicase UvrD/PcrA

hypothetical protein
MTFKLAGKAKDAYEQDGRVMVLGGPGSGKTTLALLKAKRL LSSLEPEQDVLFLSFSRAAVRQVVLRCRDVLSAPERDRISVQT YHAFCMGILRAHGRLLTGRPARILFPDREKVAKAGFEGDW NTERDRLATEESIYVFDHFASGAARLIGDVAKVAELVADRFP VIILDEFQDTSDSQWELVQALAVRSTVIILADADQRIFEYDD NVDPNRLNQVREVLKPAEFDLVGENHRSPDAGILQFADAV LRNRALPETDDVQVISVYPRALESTAHAAVRWLYSRLRKQGI QRPSIAVLARANGLVSDVSDWLSTARTYNGSELKPVAHDVL WDVELSAAAAQIVASILEWPLHDAGRAVAQTLNAVANFYD VKNAINNTPIASAVRARDSYRANADSLTGGGTVRLKAAKHL VEQYPAMSAYGGDPAADWKTARDILAGADALKEIVQAARF IRLFGATDEIGGRLSEQWDRSGGYDNAVELVRRALDQGRLV TELREPQGCVLMNIHKSKGKEFDGVLIVEAOYKGVLFDGRE EPPHMSSRRLLRVGITRARHKVIIVRPKRAPPLASP

MKACYLHIQNFRGIEDGRVSFOPHTLLVGGNNVGKSTICEA MDLVLGPERLYRRPVIDEHDFYHGKYLDEDDSPLDVRIDLVL TDLTSEERRRFGDQHLRLWDNKTDSFIDEEAGGSELADAES VEWGLPVCFIGRYDKEEDDFVGETFFCHPEPVQDELDVDQI ASLGGGRAVFRRTHKRLAGYVYLRALRTGSRALSLQRGSLLD TILQLGGEGSAEMWKDTLNHLSTLEPAIGDVPQLETVRELLR KRLGAFVNLAPGEHSAAFFASDLTRQHLREVVRLFVATQPS EHLVPYVRQGTGSINLLVFALLTIIADLKGSOSVIFAMEEPEIA LPPHTQRRVTRYVLQQMGQSIVTSHSAPVIEQFEPESIVML HRDGTKLAGAPIDLTKIKRKTYLTNRRQFAEAILARGVLVVE GSTEAVVFPAISSVLERVRAGYTHLDFAGVSMFACSGDGDV DRFGPIFTALGKKSYGACDKPNTAPSADVMANRAAFDHFW ESAESGIEHVLVRGIPVEILRKFLDAVSARGDYPAAHPYNPA MSDDDVPALAFKVLKARKGEAFAYAAVLIEQCETEADLPPE LITILITIDDELRAEPEEPIEPESASESTEGE 
hypothetical protein

hypothetical protein

putative ATPase
MVDWIRLTGGEHPDVAAQLSRAGLTAADAELRISFGRIDPT RDTIIMRVIRGHLRVNDAVRQVQEFRRSQATGS

MDDKAARLLSCLAEYELTLPEDVARQLISDRLDGIAKRMRIG RQAARSYVTNDVIQSLADEVVGSRSGTDDEASNVVSLSDRR RSRRSNRR

MSTRKLPSHISQLILTDYRVKPESVVYAAFNQSQLDGSQPYV ARRIIRTSATLDVLALVPEHLQVSRRVSSRGTEVLLFSGDAET VFLEYATEQRLEVYVSASDHNHATAIANQITSRIPEAAPPKD TVGVTVWHSGARGASPSLKSIRVPSWADIRRNYPGAVAEPL NDLFTTVRPHGYGKLILWHGEPGTGKTTALRALSREWKDW CSIDYVADPERLFFDTGYLLEVIAKTSSDELQDSNTARWRLLI AEDSDEFLRVSARREAGAALGRLLNLSDGILGQGSNTLILLTT NERLDRLHPAVVRPGRCLAQVEFTRFPAAEANRWLPENHA RVSEPKTLAELIEHRDATKQIATGIAPVTNIGAYL

MTVRHIVAVGGSDAGISAALRARELDPTTEVTVVVADAYPN FSICGIPYYVSGEVTHWTNLAHRTAADLAATGMRVFTDTRA TGINVEDHTLDVLDPTGAPEQLSYDALIVGTGAISVRPPIAGI SGPDALGTVDGVHLLHSMGDTFAVMDSLQQRDPATAVIV GAGYIGLEMAEALTTRGIRVTQIEALPEVLPTVDPELGTLVH AELERNGVEVLTNTAVSAITRTDTGALSVIATHDGQTISRTV DFVLVVVGVRPDTELAADAGAELGVKGAVVVDEAMRTNLP DIFAAGDCVHTHHRLLGITWLPLGTTAHKQGRVAGENALG GQAHFAGSLGTQVVKVFDVVAARTGLREHEALAANRGWT PVSSQSSPDDHKAYYPGATPVHIRITGDLHSGRLLGAQLVG HRSAEIAKRVDTYATALYHEMTVGGLSELDLSYTPPLGSPW DAIQMAAQTWARHHQPATATAASQPGTRRNLAAPN 
dNTP

triphosphohydrolase, broad substrate

specificity, subgroup 2

hypothetical protein

hypothetical protein

hypothetical protein

hypothetical protein

hypothetical protein
MHAIIPNDPDQAAKRSTRPYAKPKSGGDARSEGQRDRDRV LYSTAWRRLGGVTQVVTPFSELPLLHNRLTHSEKVAQVARSI AEAKILSANETTQKLVQKLGGFDLDVCEAAALAHDLGHPPF GHIGEKILDDVARNKEILDLDDGFEGNAQSLRILVIGKIRSNK YEGNNPTYATIAAVSKYPWKRAGKRADHDEIIDTDSEYRKH WKKFSAYDPQYELLTQAREFLPPCISEKEIQSLEASVMDVAD DITYAVHDLEDFYLAGILDVSWIREDLEKYIRGEQKTGDVFFE LASRLGVDYSGWFNDDEMIRAAEWAERELKSGFNRRQVN YSEVEAQARKRGSDFIDTFINAIEVRDEPFWTGGPYVGLDER NWHRVQILKEITRSYVVQRPDIALLQRGQQEVLEKFVKLLYE WSENDRARLPARLKREIEIAEEQLDGGSTIGYGKDQHAPRG KNKNRAILDYICTLTDLECLQIYYKLSGIQVHRPGMVSF

MPEQERRGALINELKIVRKTGLHRLRERIDELPELSKLATATM GAGSADDIERMLRHAFTSYAEGAQGTAIGILFGLEIGRRGAR PSVLREVAAKRLGYDSVETFRKRPEYNAIAYFADLLHRLAVD ANSQKPADVNKIDHIMSLITELTIPEYWELTRRVRQLFAAGS QMS

MAQMDRQSIVRAREDFNRVREHLHQLELSRLAENAEADM REECTIHAMRNAREVDDAARHFEGGREGLAMTIREMQAA YNTGEIAHIAMRGCHR

MAALPCFEDLWNGVTCLAAAPPPVDPLIERLRQISQQLVEE KRAMDRRAQQVIRAMQAVASAAGQDGSTVQDDTSVER WVVTE

MNSSGPGHFVDRLIGWCFSILAAAIALYCAVKLLADILPALV VVVGALTLISLVVGAGIVVFRTIRNRW

MREAEADAEVKAAIADWAPDELQDEDNPSKGSDVEVGHD 
MTDQLDVPLTADSLFWQQFFFQQPFQEAKAVGLLRHWAA QDHAPQLILEARSDVTGVEYLVGSQLRHAQQVRRSVEDLVE GSIVTSYEPGDREQISTARRLQLTTTTRPLEPSDPVAAGRSIL

HALTAVQRGERMVIQIVLGPRYQPLLGPPAPNRADQPVLSK VLHGVLPEQRPDSRQALAHKLGQHGFRAALRIGVDAATAD RRRTLLLGLAAAIRTAEAPGVHLKLRPEKPREINAPRSTWSM FTRSOHLTVTEVARLTGWPVSDRDEPFPGQPPRHPKPVRPS GAVQTGERVVATANAPGAEGPVGYSVTDALRHTWVIGPN GVGKSTLLLNLIVQDLEAGRPIVVVEPKDLVRDILARVPEHRK EDIVLVDPFDVAPVGINPLDSRYRDGRQPEVVADALFGTFK AIHGDSLGPRSADILRNSLDVLARRDDASLVMLPLLLTNPGF RRSLTQHTMRDDPYAAGPFWQWFDSLSPEAIATTVAPLSN KLRPLLTKQLRAVLAQRSPKFNIROVLTENKVLLVPLQKGVIG PESAQLLGALVVAELWQAIRERAGTSEGTRTPLMVYIDEVQ DYLRLPTDLGDALATARGLGAGFHLAHQYEKQLPPAMLDA FRNNARSRICFQLRPGDAKEMVAGQSVLSVEDFGMLPAYH VYASLVRDNAVQPWASGITSAPPPKTSDPDEIRRLSRERYG QPLDDIETGFSELLSGAAKAEKSDEMGGPKRRRRQA

MRREDNHDHAGPIGDPIRLSNKPPLTSINDKSGLANDGAHT GTTPPKQTKKRRTQSHDIEALRERLSERDLAILRSVAEHQFLT VRQIEAFYFADHPSATGGRLARRALARMRNLRLLGATNRRV GGVRSGSAGMVHYIDVVGDQLLDGRSGRGSRRFFNWSQR FVHHRLAIADTHLALIEADRQAQLELVECLVEPASWRRFVGL GGARLALKADLYIEIATTPGSDFVNPWFVELDLGTESIATVLK KCRDYEAYRRTGTEQADGSGFPLVAWSMTHPDPTKAERRR LALRDAIDNDRNLPAALFRIIAPDKLIPLLTRGGAV 
DNA methylase N-4/N-6 MGDALDRLRELPDSSIDMALTSPPYFRLRNYGEDGQIGLEQ HVDQWADRLAAISAEIHRVLVPTGTFWLNLGDTYATHPSQ GADRKSLLMAPERLALRLQRAGWTVRNKVVWAKTNPMPT SIKDRLNCTYEFVYVFAKQPSYFFDLDAVRQPHRSSLRKPYV AERRDAELWRGPNSDTATGLDAIKAAGRVGHPLGKNPGD VWLLPSSSFRGAHHATFPVRLASRAIQAGCPEARCTRCRLP WRRRVIRAIGGTAIRAALVPTCECRTTSEPGLVLDPFIGSGTT AIAAEELARDWLGIELNPDFAAMAERRILDARGRRQPEEKA A

hypothetical protein

MSTPDESAKGVKTLGIRLQPDVHAQLSFIAQLREGTITDEIQ AITGHIARSKEDPELRGRADAARAEIEREAAARQKAIASLFTT EPPGSSGDSDDTAGQSRPARRGQKGDSPPEN

hypothetical protein

MSPKAKSLQAIGHVLGIPASELFAIAPWVTPQELPTIRPYLRT KYRELPPAAVQEIETYFNDVARKHGISFDPNDGPLDGEDE 
Superfamily II DNA/RNA helicases, SNF2 family
MLLEELKPGLRIAGLIPGQVVTVIFAQSHGADTVELTFKTAS GGLDQQLIFRKDENKLSVAQTGGRPFSAPASEFKLVAEAQRI TLAGLYDPMLAVATSDVRPLPHQISAVYGELLPRTPLRFLLA DDPGAGKTIMAGLYIKELILRDDIKRCLVVAPGGLVEQWQD ELFFKFGLRFDLLTNQAIDAYINDNVFETHPLLIARMDQLSR NQELQIQIQDTEWDLVVIDEAHRMAAHYFGGKLEKTKRFQ LGELLGRLTRHLLLMTATPHSGKVEDYQLFLTLLDRDRFEGK NSQNPETTGIMRRMIKEDLLTFDGKRLFPERKAETVPYELTQ LEENLYEQVTDYVREGMNRADRVGGKRKNTVGFALTVLQR RLASSPEAIYKSLVRRTQRLHRKKQEILNGTYRETEPTVDLEG LDADDYNAEEVEQLEEELLDAATASQTVEELDAELAELADLT ALAKQVRDSGTDRKWSELRTILEDHALITDNDGAPRKFIIFTE HRDTLDYLTTRIRTLIGKPDAVKAIHGGVRRGERRLITEEFTK NPETQILIATDAAGEGLNLQAAHLMVNYDLPWNPNRIEQR FGRIHRIGQEEVCRLWNIVASNTREGDVYTRLLIKIEEQRKAY GGKVFDVLGDAFHETPLRELLLKAIRYGDDPQVREKMHEVV DATVSEGLPELLKERALAADHLAEADLAELRAAMDEARARR LQPHYIELAFKAAFTRLGGRIAKRERGRYEIPHVPPQIRASKY QPIATKYDRVTFDLEHVRPESQTRADLLAPGHPLHDAVMEE TTKLLGGALNYGTVLVSSTLEEPHLLVGVIEEVCDATGASISR RFGYAFVDSYGTVSHAGPAPYLDCVAAPDTPAVTAARQLP WLAEAEDRAMSWIITNQLPGYLAEVQPRRAAELAKTRALVI KRLEGERDRLLFDSAIAAEKEHAGDKPKESSESLNRKAVDLD VRLRKRLELLDQQELMSTKPPRIITAALVLPISMLEHEIPASAP VHAKETKDVERRGVDLVLAYEKALGRQPAEQTFTNKGFDIL STTPNGDTYRIEVKARLEGATDFYVTHNEVLLGKNAVPRYRL ALVKVDPRGPEHDQVRYLDHPFATTELGDFEATGIRGDWN 
Adenine-specific DNA

MEGTLSDSAPKRKLIEVALPLEAINRESAREKSIRHGHPSTLH methylase containing a Zn- LWWARRPLAAARAVLFAQLVDDPSARPDEFPTEELQRKER ribbon NRLHKLIERLVVWESIRNEELFAEAHAEILKSTDGNPPPILDP FAGGGTIPLEAQRLGMEAYASDLNPVAVLINKALIEIPPKFR GQPPVFPGLADSEIRSWKGAEGLAADVRAYGRWARDEAE KRIGHLYPKATLPDGSNATVIAWIWARTVTCPNPACGIDMP LVRSWWLGKKKGKESYIVPTIVDDRTAPGGRRVRFEIGNDP STGPMSSNDGTVARTTATCVACESPVPLPYVRAEGREGRLR TELMAIVAEGQRRRVYLAPTALQSDAARIGRPASVPVGSLP PHGLSPDSHQTIRIYGFYEWADLFTNRQLVALTTFSDLVAVA HDRVLADGGPSDYADAVATYLGFAVSRLANKSSSTCSWDSS TKMEAVRGVFARQALPMTWDFAEANPWGGSGGDYLEDV EWVARAVERVPATRPGFAQQADARRVVMPANTLISTDPP YYDNIGYSDLSDFFYVWLRRSLRGIYPDLLSTMLVPKADELV ANPHRHDGREGARQFFEDGFKSVFASARATATADFPITVYY AFKOSESDDDGQASTGWETLLEGMIQSGWEITSTWPMRS ELSNRMLASGTNALASSIVLSLRPRRADAPTTDKRSFITALEA ELPDALRKLQQGRIAPVDLPQATIGPGMAVFSRYAAVLEPD GKHMSVRSALARINEILDRVLNEQEADFDPTSRFAIAWYRA HGYNFGKYGDAELLANARGVVVQALDRAGVLISRAGRVNL LKPNDLGPNYDVVADLHTSNWEALHHLVRVLDAEGIERAG EFLRAALGRTDGIIDADLIKELAHLLFRVAETNGWTKDALSF NGLVTSWPEIVDTAHTKTPRTSQGSFNFEEDAD 
FIG00863870:

hypothetical protein

hypothetical protein

MALSNRDRINKMFEIVAPPLDDYISLVIGQGDPAVGAAWP KLVRAKDGTSSAKTYNAHDPQVQFRMLTESNITAGFKKGW YPFNKTLGKAGESFAIELREVRNNWAHNGMFTDDDAYRAL DTGERLLRLIGAANEADEVRSMRLNLRRVTADKEDKKVLRA AVDNPEAAGLKAWRDVLPPHDDVATGNFAASEFAADLYK VAFGGEQDSGYANPVEFFQRTYLTEGLSDLIGRAVRRLSGD DNAPPVINLQTNFGGGKTHSMLALWHVAAGLPIGEFPQAT QDLLSANKYSGGNVNRVAIVGNHFSPAGIERDGTHINTTW GELAWQLGGPEAYAYVAKADADRTPPGDALAKLLAAYAPT VILIDEWVAYARSLVGRDDLAGGTFDDQFTFAQLLTEAAKA TSGVLVVISIPASESGDAADRVVAGNAEEVGGAHGLEALKR LQNVVRRVADQWRPASSTEAYQIVKQRLFTQPDAVALASI NATARAYVDMYRKYTDDFPRESRDTAYEDRIKRTYPIHPELF DRLYEEWSSLERFQRTRGVLRLMSTVIHALWVGEDASPLIM PGSVPLATGSVNSELTQYLQDSWKAIIDADVDGPNSEPARI DKDKPLFGQRSLTKRLARTVFFGAAPTIGAAHKGLETQRVFL GTAIPGDVPGNFHAALTQLADRATYFYTGSGKYWYDLQAN ITRTARDQAERLHKEDVWAEIVRRLQSQARSRGDFTGVHV CPETNGDIPDTDEARLVILHPKVAHKKGTDSPAKEFAHMAT EHRGSANRTNRNTLVYLAADEARLEELDTAARDYLGWTHV LNNAADLDLTENQKNQACQRQTTAEQTVTARLLQTFTWA LVPAQPDPGAPFIIRETKVEGQSESLAERVSRRLGNDGDLSIR QAAATIRLAINKVPQIWKDGHVSLGSLWALYCQYPYMPRL RDRTVLNEGIVDMPMHWDVEAFALATAFDKSAHRYINLWI SGDKNAAPAPTDAVLLVQPEVALKQREEEPPHGGGDKPPY GGEPGSAKPPSIDVAFPPAKKRFYGVKTLSSDKIALDFKNIAD EIISNLREPSTSLVVKIEIEATDASGFDESKIRTVSENARTLKFD MVTPGACDTVIMPIGPAATALRADTTTSFEGKRHFPAPVW AV 
FIG00828583:

hypothetical protein
MSSHRTTFNHVGLCVSDRERSRRFYEGVLGFQFWWELDPP DGPTAQLVQLPEPLGVHATYLVRDGFVLELMDYSKRQVHA GSERVMDQIGLTHISFSVSDLPGALKKVEEFGGAVVKETVTE AMAMIRDPDGQLLELLSGGWLAALPLRS

MRFTYFAAAVGIAIALLGASPAHADYSGFTRCVGGIKQLPLN EPDPQNFQRVGVIEQDLKSGVSPAAEAQKVAGMGFDQQT AATIVQCIIEENP

hypothetical protein

MHGKQIAIAGAAIVLAGCGGSGKTATTVTSAVEITKTVTVTA PPPPFAPRTVIETDGTYRVGIDIVAGTYRSGGPSPEGGSDCY WARLSSLNSTHIIDSDIGTGPQVVMIAPTDKAFLTRSCQTW HKTD

hypothetical protein MMSGHYLKTTTDPGGGRAFTENWYFTPCGDGCADMSSP ASGVSGRAMLVDGQWTLDSTEDIVCKGGVTEGNAANAHY TWDPNTLAGTVQVVQNRGVCDHPPQSYSLSFRLTKAP

Integrase

MTIRRREAKSGTRWDVEWLLPDGTKRSKTFKTLRAAKLFDG EVRSAREDGEAVDPRGGKTELRLVYKSWLASRPDLSPKVRR GYEDNWRLRIEPKFGNWPIGRILREDVQEWINGMTADGL GPRTVRWTHSVLRMTLDHAVTDKKLRGKNPAANVRFPAM GETSHVYLTAVEVAKLAELCDTASGESSRAAKQGDVVLILAY TGLRFGELTGLNVEDVDLPARRIRVRRSITQLSGRLLEGPPKS RAGRRSVPIPERLIATLERRIAGRTASEPAIVSPKGARLGLEN WKRAVGWRTRIGELGHPTMRVHDLRHTYASLARSAGAD MKLLQVTMGHASIVVTAHTYADLYDSDLDRVADALDGLGD 

VPRAHRPDPNARLPLPSMHTLQRLFLSSDYQCQTLLEDLAR RYTSGAMGEQLRAAVQRFVAAIALEQALADDPVYRRARED VERLRERLCREVERAERRDRELARLRRDAEELWRLRARVAEL ESALAGVAHPLHQAGQHDQVPVSRQQRRAAQRAARKTC

Death on curing protein, MRRGELWFAATPGGDRPVLILTRDPVADRIGAFVVAALTRT Doc toxin RTGLVSELALTAPDDRLPSDCVVNFDKFIRCRAPRSDAGSLA CLRRGYTRCVEHFERPPAAETSIRTRALISLVCLQER

Long-chain-fatty-acid--

MSISLLLEMAASTNPHRGAVVSGDARLTVDELSVLADGGA CoA ligase (EC 6.2.1.3) GVITSCAARHVAYVGTGGALLPLLLFASARAGVPFTPLNYRL SGESLRELIARLPEPLLIVDVAFAGQVAGVGALTMHSYDFITA ARTAEPVSNFIDPDDVAVMLFTSGTTSRPKAVQITHNNLTS YVTGTVEFDSAAAEDATLICVPPYHIAGIGAALSNLYAGRKM VYLRNFDERKWLELIGAEGVTTATVVPTMLARIIAVLEDKPV ELPTLRNLAYGGSKVALPLVRKALQLLPHVGFVNAYGLTETS STVAVLGPEDHRAALCSPDPAVARRLGSVGKVVPGIEAQIR GEDGSILGPGQRGELFVRGAQISGNYADIGSVLDADGWFA TNDIAEIDEEGYLFIGGRSDDTIIRGGENIAPAEIEDVLIEHPH VRDVAVVGTEDPQWGQTILAVVVPAEGTDPDPEELRAHVR KHLRGSRTPDRVVFRNELPTNATGKVLRRQLIVEFSSVTDEF A

hypothetical protein MRINRDPAIDERLVPQLARLRGRDFFYAVDTIIAGLQARLAA KSAS 
Butyryl-CoA

dehydrogenase (EC

1.3.99.2)

MVNAQSAEVADIIKAVRSFVRDHVVALEPKIEADDDIPPQL RQACAEMGLFGTAIPEEYGGLGADVETEVLLAFELGWTTPA LRAMFGTNNGIAGEVLISAGSDEQRSAWLPRLASGAAIASF ALTESEAGSDPSTMKTSARRDGGDWIIDGAKRFITNAPYAD IFVVFARTNPEVPAAQGISAFLVPKGTRGLQIGPRDAKMGQ AGEVTAPVYMDGVRVPGEAMVGGEGQGYAAAMRSLGPG RIRIAALCVGMAERLLEESLAYATTHHQSGRPIAEFQLIQAM LADSQTDIYAGRALALEAARAFDAGTDRRTGPSVAKYFCSE MVGRVADRAVQLHGGTGYMKGVTVERLYRDARVFRLYEG TSQIQQLIIAGQSVRQYRNRAGIPS

Transcriptional regulator, MPKRYGTKEKDQAVEYIIDRLLTGKLRTGDRVDRNEIARDL GntR family GLSRVPVTEAIGQLEHDGIVTSRYHRGTFVERFDPATVLEHH EVYGLLSGMASARAATYPTPRLIDKLNELIRVLRTATDSEIFE QTTWEYRRTINHEYAGPRLQAAIRTSQSFIPKAFWVNFEDN QRFMLPSYEREHAAILRHDSTAARAACEDRSMTTARVVVTE LVRRGVFESTDAVAPVDGLLSP

hypothetical protein

putative cytochrome P450 hydroxylase
MGHEEFRLLARDFIARNVTSYYDWITDGIFPAQSVPPTHQI GVMGFRDS

MNEWYYFTHRDDVLNALRTPEIYSSKKAFDMLGSPLPLVPIS YDPPEHTRFRKVLQPFFSPHALGKMMPSLQQQVIDIIDNVA AKGECDVVTDIAIPYPSQVFLTLYGLPLADRDKLVNWKDTVI ALADLPGVPGEDKLGPALELFAYLTNAITERKANPGPDILSQ VLTGEEPLDEAEAIGLSFLFVLAGLDTVTATIGFALEKLACDP ALRGQLREDPKQTNVFVEEIVRLESAAPILPRITTEEVTVGGF RLPPGSPIRLCIGAINRDGSDDISTDRMVLDGKVHRHWGFG GGPHRCLGSHLARIELTLVVNEWLSRIPDFEVKPGFTPQIEW PANTAALISLPVQWKS 
Putative GntR-family

transcriptional regulator

L-carnitine dehydratase/bile acidinducible protein $\mathrm{F}$

Long-chain-fatty-acid-CoA ligase (EC 6.2.1.3)
MTQFGVSRPTLREAFRILESEQLIEVLRGARGGARARRPDPG AAGRYTGALLQSRGTTLDDVYQARAALEESAIGLASGRGLS RQIRELEELSTRGAGLIEDPAAYAEFDVVFHHSIMALAGNTT LSVLADQLYSIIEAHNRAHLADHPAGYDVPACRTHQRGHNK LVRLLIEGDLEGAQRHWRRHLDGVQKYMIEDSRVAVVDVL SAAL

MVDDLTGFEEAVALKKPMTGVRVIEVAEYTFVPSAGAVLA DWGADVVKIENPVTGDAQRGLVSVLSRSASRQGVPFAPM MEAPNRGKRSIGLSLALSESRPAFEELIRRSDVFLTSYLPRTRA KLRIDVTDVRRVNPDIIYVAGSGFGHHGPDRDRGGYDATAF WARGGSAQSVTQIDDDSPAPMPAGAYGDNIGGLTIAGAV AAALFGRQNTGETSSIDVSLLSVGAWAMQANINLAMLYGC PLPVIGGRAPAPGNPLTGTYRCKDGRFIQLSMLQPTRYWPE FCSLFGLASAATDPRFASMEALAQHNDEAIRLVAEAIGSRPF EECNRLLDQSSGPWAPLQDAWEVANDEALIANGRIADIIDS QGHPQRLVANPAQFDGAPTRLNRAPLFAEHTDEVLRDLGV DETELLQLKYAGAIT

MEPAPAPPRQFATWSQRILHHLLSDSDRDAIVSMNHERIES WTRAELVARTAGASDYLDVHGVQAGDCVPALLTSQPHSVA LLIAGALTRRPLAPLAPRVTHREVFASLKNISGPVLVTEPQYR DFASELAKATGKRIAVVDEIGAGNVALGADARRPEDIACML HTSGTTGLPKPVPVPEGPFERRAEILGQLCGFNGDARYVTA ALFHHVAGLGNLMPALANGASIILIPPLSVDLWRNLASAAPT HTTLVPSVIEMLLEEGVPAPPSLRVLAYGGSSIHPDTVRRMQ KVMPAVDLINLFGQTEGSPVTVLNADDHRRAAAGQEGLLS SVGRPAPGVELRLNEPDANGIGEIWARSDHSFVVDEQGW QRTGDLGRVVDDFVYLVGRRGDKIIRGGENVFPLEVEQILET HPDVVSAGVVGKPDRRLGETIVAFVVPVDVEEPPDSENLRV HCRAQLAGFKVPVEWIFVDRLPRNPNGKLLRGELSRELARR TSCGAKGHRVIWQ 
Alpha-methylacyl-CoA racemase (EC 5.1.99.4)

hypothetical protein

Alcohol dehydrogenase (EC 1.1.1.1)
MGPLRGTRIIELAGIGPAPFGCMLLADLGAEVVRVERAMSA AALASIDDGGRSQADPRRYIPHRGRRSISLDLKSAEGRDALL RLVETADVFVEGFRPGVTERLGVGPDDCLARNSRLVYARM TGWGQSGPLASTAGHDINYIALAGVLDNFRRTGERPMPPL NLVGDMGGGGMMLAFGITAALVHAARTGEGQVIDAAMV DGAALQMAVVLGGRAQGRWPGEPGSNYSDTGAPQYEVY ECADGKFVAVGALEGPFWSEFLRILDQPAGAVPSRDDPAL WPQGKEALAKVFRTKTRDEWAAMFDGSDACVTPVLSIDEA PAHPHAKARSAFITVGGIEQPAPAPRFSATPADVPSAPPDR GEHTEEVLRELGLTDEEIVAATGSQRV

MTETGSVVVGYSPPPRTDKPLKVTDFVRYQGASGDMNPIH HDPEYAARAGYDQVFAVGMLAAGRLATYVTDWLGPENVR KFGVRFKEQAWPGDELTYTATVVDSRTDENERVVDLDLVC TRHTGGVHLTGWATVALKTPTTDETDDGGPPSSD

MAEPFDIPIELGKSREFAKATGSKHPAHYSPGGVTPPTFLMT AILWQGPDNNAWPKDRDMRRVLHASQEFAFPKGPPRIG MQLTGQQRIEGSHTKEGKRGGTLTFTTLLTEFRDEKGDLVA EVRNTFVETSKPTGGDH

MMKATALVLETPNHLIERHIEIPAPAAGEAILRVLACGLCGS DHELFTGAMPGSLPLIPGHEVVGVVERATDEFLSARGLEQG EVVALEVFORCEQCDACRRGAYPLCRTYGLARSYGNTSIEW GSGLWGGFATHLLLGCDALVHRVPPGLDAAYATLFNPLGA GVRWAHVLPQVQQGDVVAVLGPGLRGLSSVAVVSIAGAA FTLLTGAGSRDRERMELGRTLGATETVDVTTTDARALLKERT GGLADVVVDVTAAAPAAFLQAIDLARPGGTVVVAGTRGLH ALKDFNPDRFVLKELTLLGARGVDGTAYARALELLATDDRFE AIPRVTCALDAGSVADLLGDMAHGEAPPLHAVIVP 
Putative uncharacterized MSEDEQRERHGKSLRFMFTKVPFNKVVGLELIDWSEPDVA protein BCG_1584c

VVRLPFSEAIDNSGGTAHGGAIATLADVAGSAAAWNGHDY EKGTRGATVSVTINFTGAARGEAVIATARCVRRARELNFTEV SIVSESGRPVASGTVIYRIAT

3-ketoacyl-CoA thiolase (EC 2.3.1.16) @ AcetylMPEAVIVEAVRTAIGKRNGALSGVHPTTLGAVVLGELVART 2.3.1.9)

GVDPADVDDVQWGCVTQLGDQSSNVGRFTVLAAGWPET

\section{MSRVPLGAGRATGMPYGPAAYDRFPGADFNQITGAQAIA EKWGLSRRRLDEYSSRSHELSAEAADHGKFDEQIVAVETSD} GHFRVDEGLRRGTTPETLAKLSSVSGPDGVIHAGNSSQISDG AAAMLVTTPEKAKSLNLRPLARYVRGVAVGADPRLVLTGPI PATQQVLAKSGLTIGDVGAFEVNEAFAPVPLAWLAETGAD PARLNPLGGAIAVGHPLGASGAILMTRLVHHMRDKGIRYGL QTMCEGGGTANATIVELFGD

Enoyl-CoA hydratase (EC MPELLSDAEAGVLTLTLNRPQSRNAVSYSLLDNLIEAFEAAD 4.2.1.17) SDPSIRAIIVTGSGDSFSYGTDLAAGGGGLDVNAPGFKPLRG TKRDVGGVLALRLFSCTKPVIAAVNGTAVGVGVTMILPMD VRIAADSARFGLPFTRRGIVPESCATWFLPRIVGIATAIEWCV TGRVFDAQEAAARGLVSELLPAEQVLPRAREIAAQIAEQASP LSVALTRQMLWRQLGSPHPMSANRLESQAFLSLGGSADTK EGIAAFKEKRKPHFTTAVPDDLPAFYPWFADEEFADKS 
3-ketoacyl-(acyl-carrierprotein) reductase( EC:1.)

MRLDGKVALVTGASRGIGLAIANCYAVNGAKVMLASRKAD GLAAAAATLDGQVAVKVANTRNLDDIEGLVDAVLDRWGR LDVLVNNAATCPYVGPVVDVEPGQRDRNFEVNLRGSLFLIQ KAWRAWMVSNGGSIINISSVGAFRTHRTMGFYDIGKAALA QLTRHLAGELGPRVRINAIVPGLVETRMAESIIKTRGVDIAAR IPLQRNGIPEDIAGAALFPAGAAWSWMTLPRRHAKGAIEP DRLAVDVGVLEQFARHAAELLRSSQPSRVRDVGCEGRTHFV TESSEHRGIEDPWRDCVHPDALRRQIARQDECHSAHTGFR GRIGRLTNLTLERSGRRRHHHGAPVAIGRRWIARDSFSREP DDVVAADEIHSDDFFETVQRVCVSVTVEYTQPIAAPSRAMY DRPQRSCINRGVERLTQTLGISDIRRDKGCADVLGGSRAVR RREVGDNHAGATPRQRRRTRESQPRRSSGHQGSRVIQIHL GLLYTSRQWIHINVKHLLARVDGTKSDRIVAGRRGVVLADS NLWLNPTAGGRRGWIPRHELTGEAPEHAHA

putative acyl-CoA

MGGWLSANAESLDEFRHHRAEAAEERWARDLRFIRVLYAA dehydrogenase( EC:1.3.99.- GWNRHGWPAEYGGVGGPAVLRNVLYEELENAGYRVPDC ) VIQLELQGEAFMKFAPEIAAARIPAALRGDEMWTQGFSEPE AGSDLASLRCKAVQDGDEWVITGQKTWSSYALSARWMGV LTRTGTPESRHRGITMFLVDLESPGVEVRPLRLANGVDECA DVFFDNVRVSADNRIGEVGGGWDVAMYLLQFERANYGW LRQAHLARRLRELVSNIDAPDRHTANVLGNCWLANLALRLR CGDTVRRLFAGQRIGPEASIDKVLIAQAEQPLNDAFRDLFPA QFLFDPGEESEVWRSEWYYSRAASIYGGAGEIQRGIIADRLL GLPKEV

hypothetical protein MVQTKSTELFEEDGRNSPVFVKHIFKANVTGHRSRVAAVSS 
acyl-CoA dehydrogenase domain protein CoA ligase (EC 6.2.1.3)

hypothetical protein
MRGVDGDSVSSILLLAGEEAVLVPTSGLQCSPAKGFDIVDG WTRWRGSVSADDSDVLSTDGARHVRAVVRAALASELNGIA SKVNELAVDHVTSRHQFGQALGSFQSVRHRLAETHVAIHAA MPVIELAWQATACGDAGSAELATAAKALAGRAFEIAAKNA HQVCGGMGLSWEHPLHRWVRRGTVLNSLIGSPDELAADL GGMLARGAALPVPNALCDDENOSTPA

MRRRESVHPCMTRQPGVEVDPRHDGVTPLGENVDSPSHH YVTSLRHFAVKRPQDLAIVTPARSVTFAELSERANSLAGYLHS RGVAAGTPVALMSANSAELIEAFYAALVLGAPPANVNPRYH AHEVRYVLENCAAGAVLFDPTSATVVAEAVSAMEEPPFPLE FGSSRWRAAISSTTDYVRPQSVDDRLLIYTGGTTGLPRAVE WRVVDHFRMIWOMVKPTEPPPTPEALIDSGRRAPTASPCS PILHGVGLSLTLNTLNGGGTVVVSDRLTFDAADTFDLVRRYD VAVLGIVGDAFARPMLDELETGRWNGKLPSLRAISSAGAS WTSSIRDRMANLLPGVKMVSNFGSTEALVARDISSDHSIDP GDGLVVIGENDRPAQPGEVGVVATAGYLPLGYLGDPQKTA ETFRTINGRRYAVTGDEARVEADGRITLLGRGSAVINTGGEK VWPDEVEAVLRSVPGVLDAVVVGRPDERWGQRVAAVLRI AGTAEISDQRLSQGCRERLAPYKCPRQYVRVDEIPRTPVGKP DYRAIDSLLEES

MPKRYGTKEKEQESAAREERSTMMGRVAVTELVNRGGSE TYRGRHARYRDLDTELVGGY 
Butyryl-CoA

dehydrogenase (EC

1.3.99.2)

MRRTLFDQEHEEFRVLVRDFIAKRVVPHFDEWVDEGIVPR QLYRELGAIGAMGFAIPEQYGGGGVDDYRYNAVLQEEAAR ANVTLGTMRTHLDVVLPYLLKYANDEQRERWFPGLVSGDL FLAIAMTEPGTGSDLAGVKTTAKRDGSDFIVNGAKTFITGAA HADLIIVLARTGEPIDGNRRSGLSLLVVEDGVAGFTKGRRLP KLGLRVQDTVELSFEDVRVPAINLLGEVDQAFSYLGHNLAQ ERLAIAVGSVAQSRAAMEMTIDYVKNRNLFGTTVSAMQNT KFEIAAVATEIEAAQAMCDNGVRDLVDGELSGADAAKIKLF CTEMQARTVDRCLQLHGGYGYILEYPISRLYADARVSRIYGG TSEVLKSIISKSLGL

Enoyl-CoA hydratase (EC 4.2.1.17)

hypothetical protein

MARWSDEFVFGQVWTDSDIPWEQQILVAITALATLGHHT QLRNYLHGALQGGVSEPALRHALSMLTVYAGFPVAIQALN VLSEVVAREARTTTTHPENAPGSASGR

Acyl-CoA thioesterase II (EC 3.1.2.-) VAANMTVTDDRLPHSLHCTYLAAGDPAHGLEHQVTRIRDG RSFSVRRVDVFNGGRLSVSATVSYQTKSAGVEHRRTAPRVP CPDDLPTFHGASNAAWIPWAEENPELEMRVAPHDPGDSL GRRTFWLKIRHDPAIGSADDLLEAAYAAYASDFTMIASIRLP HEEPDVKTHVMTTLTHSLYFHKPFAASQWHLVDHWSPAA AGGRGLSIAHAYNASGDLTMTAVQESLVRSASKKAG 
probable

oxidoreductase/Short-

chain dehydrogenase

putative cytochrome P450 hydroxylase

hypothetical protein
MKIIITGASSGIGKATATALAARGHQVVIACRTLKKGEEAAA QMSGDVRVQHLDLADLASVRRFADAVDTVDVLVNNAGVL GLPYTRTVDGFEAHMGTNHLGHFALTCLLADRTTDRVVSLS SSAYKYATPHLDDLNWERRKYSKSQAYGESKLATMLFINELA RRGVRAYAADPGIVFSDITRDGGAILRWSGKYMHPIIGQTT GNGARSTLLAIETTAPSGTCFAPGGLMHQWGKPKVVIPHR KARNPRTALRLWELSAELTGCDAPYEPQASE

MTETAVGKPDVRYDPYDFEIDNDPYPTFRRLRDEAPLYYND EYDFYALSRFHDVKQASVEWETYPSGRGSVLELIKSGAAIPP GFILWEDPPRHDVHRGLLARVFTMRRIAQIEDKVRTVCTAT LDKFVDTGRFDFVADLGSEIPMRTIGMMLGIPDSDQVSHR DRTNEGIKLADGQAPVDGSALLTLTDTQRIGDYINFRRRHP ADDLMTDLLTATCTDSGGHERQLQDSTIQTYVGLLAAAGN ETTVRLFSWAGKVLAEHPDQRRELANDPSLIPAAIEELLRFE APSPVQARYVARDVEHHGRTMPQGSVVLLLTASANRDERA FEDPDRFDIHRKTKNHVSFGFGIHHCLGAHLARLEARVGLEE VFKRFPEWDVDWDNAVQSHTSTVRGWEKLPVVASPSLRD $\mathrm{R}$

MAFVTGAARGQGRAHRFPTSSPEEVANLAAGEARYITGQQ IRIDGGALIEFPNGPTRI

MKLPVEPELESLRAAIAARAKSTVRPLAAQVDREQKFSWEL WAAVRELGLSRIHFPEDHGGDGGTFRAYTIASTEPAEYCAV ASLYPGTSIQVAMALLQHGNPEHSRRFFAFWAAKRCGLGL YRAVDRCGFSAQRVAFIPGYMSARQAAAVDPEAPTRPTSR WLRSSSSLSWNFPAQTRRKIPARQLRPPMLWPSRR 